Journal of Thermal Engineering, Vol. 7, No. 2, Special Issue 13, pp. 256-269, February, 2021

Yildiz Technical University Press, Istanbul, Turkey

\title{
THREE-DIMENSIONAL SIMULATION OF A TURBULENT FLOW AROUND A TAPERED CUBE DUG IN THE MIDDLE
}

\author{
Lamia Benahmed ${ }^{1,{ }^{*}}$, Khaled Aliane $^{1}$, Ali J. Chamkha ${ }^{2}$
}

\begin{abstract}
To analyze the influence of the tapered form of the two upper vertices of a rectangular cube placed in a channel and the impact of the insertion of hollow in the center of the obstacle, a three-dimensional study was executed using K- $\omega$ SST turbulence model. Different models of the form of the cube were presented to examine the features of the flow with a Reynolds number $\mathrm{Re}=4 \times 10^{4}$. The turbulence kinetic energy, 2D and 3D time-averaged streamlines, trace-lines, streamwise velocity profiles, pressure profiles were obtained using the ANSYS CFX calculation code and the finite volume method were employed for resolving the governing equations. The streamlines showed in the model of the tapered cube with hollow a formation of another vortex downstream of the cube at the outlet of the hollow. For the streamwise velocity, there are two recirculation zones: one logarithmic zone due to the main flow, the other is a lower parabolic return zone due to the recirculation vortex.
\end{abstract}

Keywords: Turbulent Flow, Tapered Obstacle, Hollow, Finite Volume, Turbulence Models, ANSYS -CFX

\section{INTRODUCTION}

The study of atmospheric pollution around buildings has become a necessity given the even increase in the rate of pollution in cities [1]. This pollution becomes very dangerous for the health of the inhabitants especially if it remains stagnated and blocked by the buildings. For several years now, many experimental and more recently numerical studies have concentrated on the understanding of complex flows around various more or less profiled models. This type of flow is generally found in many industrial applications, such as the cooling of electronic components [2], atmospheric flows around buildings [3], fins of turbomachines, etc. Several studies have been carried out on the application of flows around obstacles, Hussein and Martinuzzi [4] presented an experimental study of a three-dimensional circulation in the canal which contains a cube. The results show that the development of the turbulence dissipation rate from the recirculation zone to the asymptotic slip. Martunizzi and al. [5] studied the flow around obstacles prismatic with different aspect ratios using the techniques of crystal violet display, oil film, and laser. The results showed a nominally two-dimensional region exists behind the obstacle and upstream of the recirculation zone. Hwang and Yang [6] have carried out a numerical study on swirl (vortex) structures around a cube inside a canal. They found out that the number of vortices increased as the Reynolds number raised. Filippini et al. [7] investigated the instance of the current around cubes placed into a channel, using the Large Eddy Simulation (LES) model. The results obtained showed that when the ratio S/H increases, the average drag coefficient augments for the second cube while it remains approximately constant for the first one. Chang Lim et al. [8] and Krajnovi'c et al. [9] provide using the standard Large Eddy Simulation (LES) model a numerical simulation of the flow around an area contains cube, inside in a turbulent boundary layer, and comparing with experimentation. In the same approach, Dan Gu et al. [10] and Becker et al. [11] to study the structure of the flow around three-dimensional obstacles, an experimental simulation has been presented using different aspect ratios, in two different types of boundary layers. The experimental results showed that the flow structure around the obstacle depends on its aspect ratio, the angle of attack, Reynolds number, and type of boundary layer. Yakhot et al. [12] presented a study of flow around a cube mounted surface with Direct Numerical Simulation (DNS) for a Reynolds number Re $=5160$ by using the immersed boundary method. The results support to employ immersed-boundary methods for simulating complex turbulent flows. Rostane et al [13] studied the impact of the curvature of the below edge of a block inside in the canal on

This paper was recommended for publication in revised form by Regional Editor Erdal Çetkin

${ }^{1}$ Department of Mechanical Engineering, University of Technology, Tlemcen, Algeria

${ }^{2}$ Department of Mechanical Engineering, Prince Mohammad Bin Fahd University, Al-Khobar, Kingdom of Saudi Arabia

*E-mail address: benahmed_lamia91@yahoo.fr

Orcid id: 0000-0002-1718-0015, 0000-0003-1836-4593, 0000-0002-8335-3121

Manuscript Received 18 May 2019, Accepted 11 September 2019 
aerodynamic phenomena, in particular, the vortex behind the cube. The authors studied the influence of three radius of curvature $\mathrm{R}=0.2 \mathrm{H}, \mathrm{R}=0.3 \mathrm{H}$ and $\mathrm{R}=0.5 \mathrm{H}$, for $\mathrm{Re}=10^{5}$. They concluded that the dimensions of the vortex downstream of the obstacle decrease by decreasing the radius of curvature. Sari-hassoun et al [14] studied the influence of the curved shape of the lover's edge of a rectangular obstacle. They compared two obstacle models using a qualitative approach, where they analyzed the dimensions of the recirculation zones, the velocity field, and the kinetic energy and dissipation. Aliane et al. [15] Performed a numeric analysis of flow around a block placed in a rectangular channel, using two models of obstacles: a rectangular cube, and the other rectangular with upstream round edge. The turbulence model used was k- $\varepsilon$. Salim et al [16] studied an approach to treat turbulent flows on a surface-mounted cube using the $\mathrm{y}+$ wall as a guide to select the appropriate grid pattern and corresponding turbulence models. They used the Fluent calculation code. The study is divided into two parts: the first part and the second part, deal respectively with the low and high Reynolds number. Heguehoug et al [17] presented a study of turbulent flow, stationary, three-dimensional, incompressible, and without heat transfer around an isolated 3D profile and through a series of 60 blades constituting a fixed wheel similar to that of a turbo machine. Merahi et al. [18] have contributed to the study of stationary three-dimensional flow and incompressible through a cascade of dawn by a digital simulation based on the k- $\varepsilon$ model, and it was concluded that losses due to the change in the angle of incidence are considered to be the main cause of the decline in of turbo machinery. Amraoui and Aliane [19] presented the study of fluid flow and heat transfer in a solar flat plate collector by using Computational Fluid Dynamics (CFD) which reduces time and cost. Sercan et al. [20] investigated the flow characteristics around a surface-mounted cube at $\mathrm{Re}=3700$ in terms of Computational Fluid Dynamics (CFD) and then compared with experimental results using three different models of turbulence. Kanfoudi et al. [21] large eddy simulation (LES) have been employed to present a numerical analysis of the turbulent flow structure induced by the Cavitation Shedding. Djeddi et al [22], have simulated a viscous fluid flow on an unconventional diamond-shaped obstacle in a confined channel is simulated in low to moderate Reynolds numbers. The diamond-shaped obstacle is geometrically modified to represent different blocking coefficients depending on the height of the channel and different aspect ratios based on the length /height ratios of the obstacle. The simulations are divided into two stationary and unsteady flow groups. Liakos et al. [23] performed a Direct Numerical Simulation (DNS) of steady-state laminar flow over a cube at Reynolds numbers ranging from 1-2000 based on the cube height. In the same work, Diaz-Daniel et al. [24] presented a Direct Numerical Simulations (DNS) of a wall-attached cube immersed in laminar and turbulent boundary layers at various Reynolds numbers $\mathrm{Re}_{H}$ ranging from 500 to 3000. Additionally, Sumner et al. ([25], [26]) assessed the effect of the aspect ratio and the incidence angle of the flow above the free end of a surface-mounted finite cylinder and finite-height square prism and Siddhesh Shinde et al. [27] presented a large-eddy simulation of flow over a wall-mounted cube-shaped obstacle placed in a spatially evolving boundary layer in order to understanding how variations in the cube height h modify the flow dynamics for the situation where the cube is within the boundary layer. M. Ennouri et al. [28] studied the modeling and simulation of the flow inside a centrifugal pump through non-cavitation and cavitation conditions using an SST-SAS turbulence model. Sari-hassoun et al [29] affected an experimental study of flat plate air solar collector. The conclusion obtained was that the air temperature at the outlet was different from that inside the collector. M. Bayareh and A. Nourbakhsh [30] studied the effect of different geometries of inner and outer tube surfaces on heat transfer of a double pipe heat exchanger is studied. Two-equation standard $\kappa-\varepsilon$ turbulence model is used to model the turbulent flow. Simulations are done for different cases include convex, concave and smooth surfaces for inner and outer tubes at different Reynolds numbers. Results show that the maximum heat transfer corresponds to the convex-concave case in comparison with the smoothsmooth one.

To examine the influence of the tapered form of the two upper peaks and the impact of the hollow in the middle of the obstacle to check the detachment length, the structures of the vortex, the recirculation zone, threedimensional simulation of flow around a cube inside a horizontal channel has been carried out. Three different models of the cube (simple cube, tapered cube, a tapered cube with hollow) were processed to execute this.

\section{MATHEMATICAL MODEL}

Averaged equations of conservation of mass, momentum, energy, and Reynolds are: 
Journal of Thermal Engineering, Research Article, Vol. 7, No. 2, Special Issue 13, pp. 256-269, February, 2021

- The mass conservation equation

$$
\frac{\partial \rho}{\partial t}+\frac{\partial\left(\rho U_{i}\right)}{\partial x_{i}}=0
$$

- Momentum Conservation

$$
\frac{\partial U_{i}}{\partial t}+U_{j} \frac{\partial U_{i}}{\partial x_{j}}=-\frac{1}{\rho} \frac{\partial P}{\partial x_{i}}+v \frac{\partial^{2} U_{i}}{\partial x_{j} \partial x_{j}}
$$

- $\quad$ Energy Equations

$$
\frac{\partial \bar{T}}{\partial t}+\bar{U}_{j} \frac{\partial \bar{T}}{\partial x_{j}}=\frac{\partial}{\partial x_{j}}\left[\left(\frac{\mu}{\operatorname{Pr}}+\frac{\mu_{t}}{\operatorname{Pr}_{t}}\right) \frac{\partial \bar{T}}{\partial x_{j}}\right]+\frac{1}{\rho C_{P}} \Phi
$$

- Reynolds Equations

$$
\rho \frac{\partial \bar{U}_{i}}{\partial t}+\rho \bar{U}_{j} \frac{\partial \bar{U}_{i}}{\partial x_{j}}=-\frac{\partial \bar{P}}{\partial x_{i}}+\frac{\partial}{\partial x_{j}}\left(\mu \frac{\partial \overline{U_{i}}}{\partial x_{j}}-\rho \overline{u_{i}^{\prime} u_{j}^{\prime}}\right)
$$

The turbulence model used in this study was the K- $\omega$ SST (Shear Stress Transport) model of Menter [31] is derived from the Standard k- $\omega$ model [32]. This model combines the robustness and accuracy of the formulation of the k- $\omega$ model in the near-wall region with the k- $\varepsilon$ model [33] and all its types for the free flow away from the wall.

The definition of the turbulent viscosity is modified to take into account the transport of turbulent shear stresses.

The formulation of the two-equation model is:

$$
\rho \frac{\partial k}{\partial t}+\rho \bar{U}_{j} \frac{\partial k}{\partial x_{j}}=\tilde{P}_{k}-\rho C_{\mu} \omega k+\frac{\partial}{\partial x_{j}}\left[\left(\mu+\mu_{t} / \sigma_{k}\right) \frac{\partial k}{\partial x_{j}}\right]
$$

Specific dissipation rate:

$$
\begin{aligned}
& \rho \frac{\partial \omega}{\partial t}+\rho \bar{U} j \frac{\partial \omega}{\partial x_{i}}=2 \alpha \rho S_{i j} S_{i j}-\beta \rho \omega^{2}+\frac{\partial}{\partial x_{j}}\left[\left(\mu_{t}+\sigma_{\omega} \mu_{t}\right) \frac{\partial \omega}{\partial x_{j}}\right]+ \\
& 2\left(1-F_{1}\right) \rho \sigma_{\omega 2} \frac{1}{\omega} \frac{\partial k}{\partial x_{j}} \cdot \frac{\partial \omega}{\partial x_{j}}
\end{aligned}
$$

The blend function $F_{1}$ is defined by:

$$
F_{1}=\tanh \left\{\left\{\min \left[\max \left(\frac{\sqrt{k}}{C_{\mu} \omega L}, \frac{500 v}{L^{2} \omega}\right) \frac{4 \rho \sigma_{\omega 2} k}{C D k \omega^{2} L^{2}}\right]\right\}^{4}\right\}
$$

Here y is the distance to the nearest wall. In the near-wall region, $F 1=1$, while it goes to zero in the outer region $C D k \omega$ is given as:

$$
C D k \omega=\max \left(2 \rho \sigma_{\omega 2} \frac{1}{\omega} \frac{\partial k}{\partial x_{j}} \cdot \frac{\partial \omega}{\partial x_{j}}, 10^{-10}\right)
$$


Journal of Thermal Engineering, Research Article, Vol. 7, No. 2, Special Issue 13, pp. 256-269, February, 2021

Eddy viscosity is given by

$$
v_{t}=\frac{\alpha_{1} k}{\max \left(\alpha_{1} \omega, \sqrt{2} S_{i j} F_{2}\right)}
$$

The second blending function is defined by:

$$
F_{2}=\tanh \left[\left[\max \left(\frac{2 \sqrt{k}}{C_{\mu} \omega L}, \frac{500 v}{L \omega^{2}}\right)\right]^{2}\right]
$$

To prevent the accumulation of turbulence stagnation regions, limited production was used:

$$
\begin{gathered}
\widetilde{P}_{k}=\min \left(P_{k}, 10 . C_{\mu} \rho k \omega\right) \\
\boldsymbol{P}_{k}=\mu_{t} \frac{\partial U_{i}}{\partial x_{j}}\left(\frac{\partial U_{i}}{\partial x_{j}}+\frac{\partial U_{j}}{\partial x_{i}}\right)
\end{gathered}
$$

The model constants are calculated using the mixing function F1:

$$
\phi=F_{1} \phi_{1}+\left(1-F_{1}\right) \phi_{2}
$$

The values of the model constants are:

$$
C_{\mu=0.09,} \alpha_{1=5 / 9,} \alpha_{2}=0.44, \beta_{2=0.0828}, \sigma_{k 1=0.85}, \sigma_{k 2=1.0}, \sigma_{\omega 1=0.5}, \sigma_{\omega 2}=0.856
$$

The k-w SST model is mainly recommended for applications such as fluids undergoing sudden changes in stress, flowing in curved surfaces or cases of boundary layer separation, so is the perfect model for our simulation.

\section{MODEL DESCRIPTION AND COMPUTATIONAL DOMAIN}

The obstacle used in our problem studied (Fig.1) represents a cube that has tapered upstream and downstream edges, mounted inside a horizontal channel of length (L) and height (h). (Fig. 2)

To obtain a better precision of the results, it is necessary to generate a mesh well refined. It opted for a structured hexahedral mesh in our case using calculation code ANSYS CFX. Fig.4 shows the grid of the mesh used.

\section{Boundary conditions}

Since the flow is turbulent, the model of turbulence k-w SST was chosen to analyze the problem more precisely. All "walls" are adiabatic and have no-slip conditions. According to the models selected previously, equations had to be solved using the following factors: The incoming flow velocity $\mathrm{U}_{0}$ corresponds to Reynolds number $\operatorname{Re}=4 \times 10^{4}$ $\left(\mathrm{Re}=\mathrm{U}_{0} \cdot \mathrm{h} / \mathrm{v}\right)$ and the channel height (h). The height of the obstacle is $\mathrm{H}=25 \mathrm{~mm}$ and the channel height is $\mathrm{h}=2 \mathrm{H}$. The velocity is zero $\mathrm{u}=0 \mathrm{~m} / \mathrm{s}$ near the lower and upper walls of the channel and above the obstacle. The pressure at the outlet $\mathrm{P}_{\text {out }}=0$. The boundary conditions of the problem treated are given as follows, as shown in Fig.3:

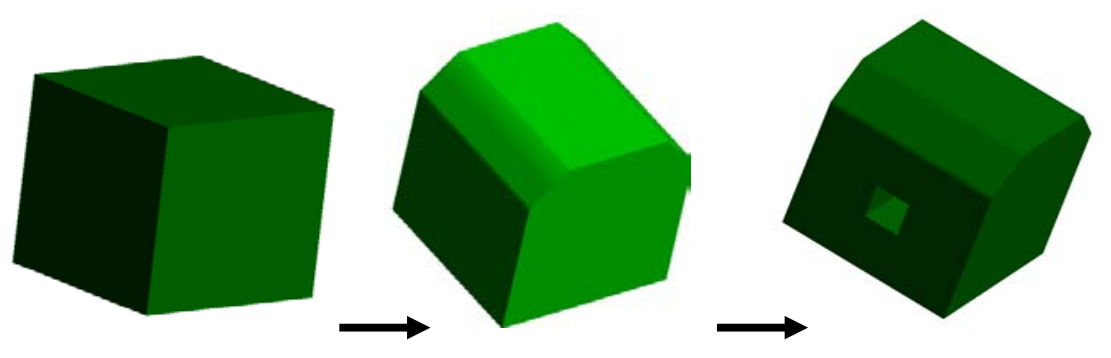

Figure1. Model of the body (3D view) 

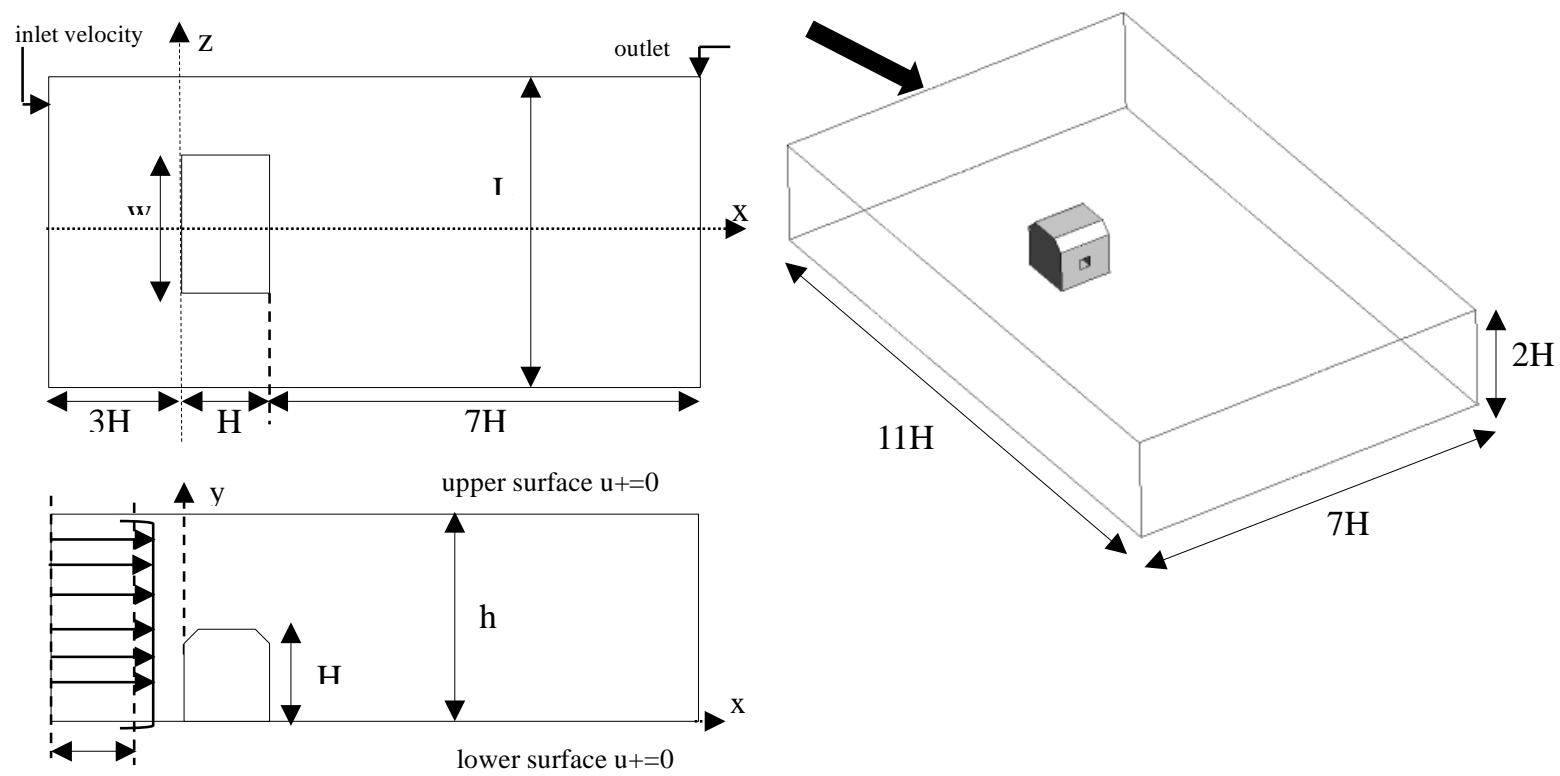

$\mathrm{U}_{n}$

Figure 2. The geometry of the computational domain
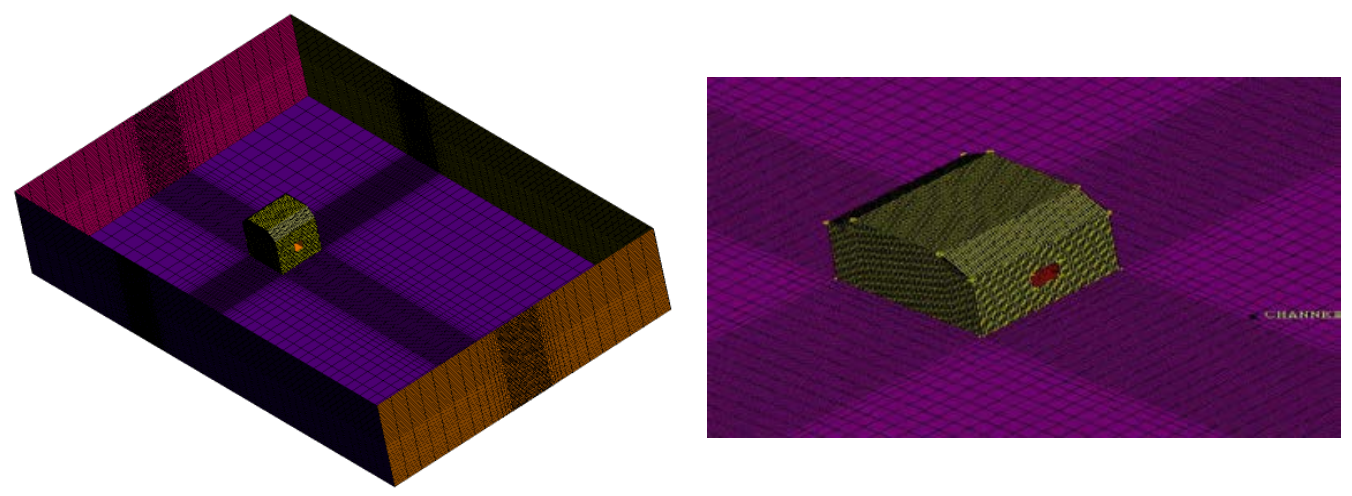

Figure 4. The grid mesh of the configuration (cube tapered with hollow)

\section{RESULTS AND DISCUSSION}

An initial study of the grid independence was executed by a serial of test simulation in the computational domain corresponds to the velocity. For this, Many meshing sizes were tested (Fig.5) to ensure the grid independence of results, including 685020, 865274 and 987100 hexahedral elements. The values in Table.1 indicate the properties at $x / H=0.5$. This study proves that there are comparatively low differences between the three grids. The mesh size of (865274) was chosen as the best solution between precision and calculation time.

Table.1 Different meshing sizes

\begin{tabular}{|l|c|c|c|}
\hline & L/H & h/H & Grid \\
\hline Configuration 1 & 7 & 2 & 685020 \\
\hline Configuration 2 & 7 & 2 & 865274 \\
\hline Configuration 3 & 7 & 2 & 987100 \\
\hline
\end{tabular}


Journal of Thermal Engineering, Research Article, Vol. 7, No. 2, Special Issue 13, pp. 256-269, February, 2021

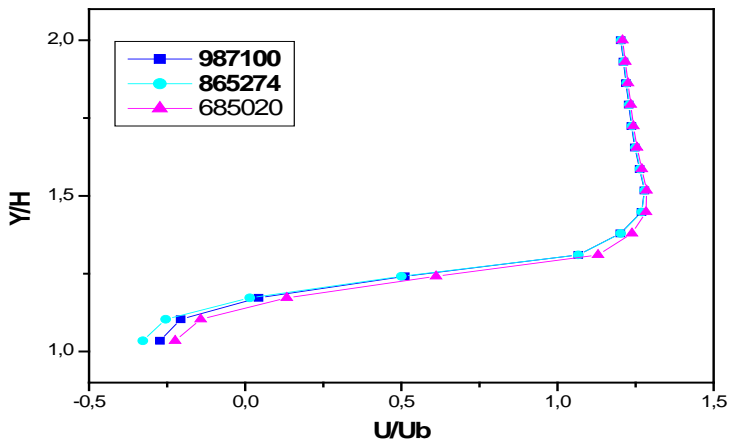

Figure 5. The grid sensitivity

In this study, the flow domain experimentally carried out by Martinuzzi and Tropea [5] was prepared to validate the flow characteristics around the cube of height $\mathrm{H}=25 \mathrm{~mm}$ placed in a channel of height $\mathrm{h}=2 \mathrm{H}$. Figures (6) presented the profiles of the axial speed on the plane of symmetry $(z=0)$ and at different positions located on the obstacle $\mathrm{x} / \mathrm{H}=0.5$ and $1(0<\mathrm{y} / \mathrm{H}<2$, upstream $(\mathrm{x} / \mathrm{H}=-0.5)$ and downstream $(\mathrm{x} / \mathrm{H}=1.5)$ of the obstacle $(1<\mathrm{y} /$ $\mathrm{H}<2$ ). These speed profiles show, compared to those obtained experimentally by Hussein et al [4], shows an almost perfect coincidence and a good agreement between the two approaches.
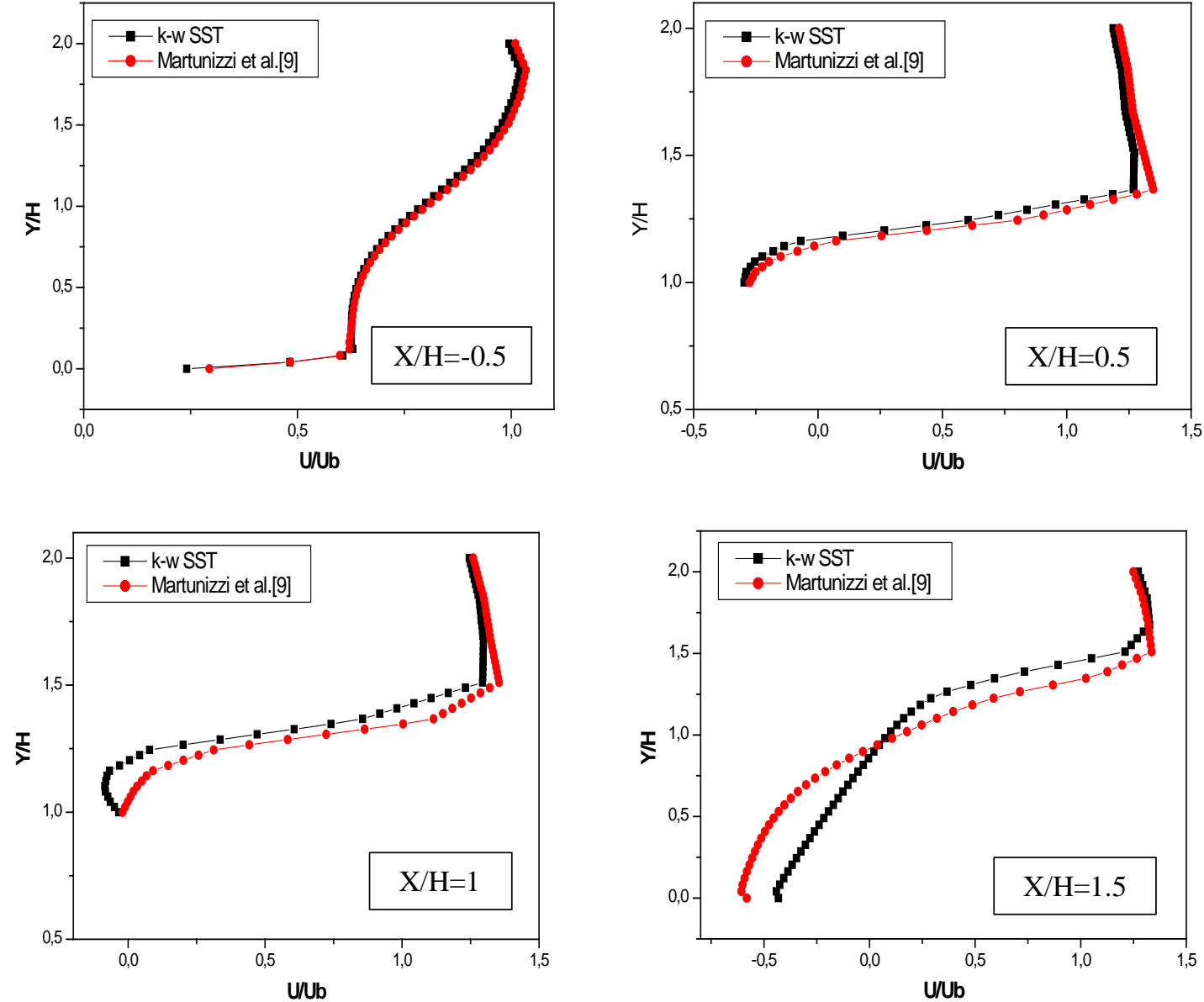

Figure 6. Validation of the velocity profiles at different positions in the plane of symmetry and for $\mathrm{Re}_{\mathrm{h}}=$

$$
4.10^{4}
$$


The study of the influence of the tapered form of the two upper peaks and the insertion of a hollow in the middle of a rectangular cube has been examined. A three-dimensional study was carried out using the ANSYS CFX calculation code. Turbulence model K- $\omega$ SST has been employed to examine the features of the around the inclined cube at Reynolds number $\operatorname{Re}=4 \times 10^{4}$. The turbulence kinetic energy, 2D and 3D time-averaged streamline on the symmetry plane $(\mathrm{z}=0)$ and at the floor of the channel, streamwise velocity profiles, trace-lines around the cube have been presented.

The variation of contours of the turbulent kinetic energy in the symmetry plane $(\mathrm{z}=0)$ for various block models (simple cube, tapered cube and tapered cube with hollow) is illustrated in fig.7. It is observed that this energy is maximum and higher downstream of the simple cube, and at the outing of the hollow, it is minimal and almost zero at the tapered edges of the cube. It can be noted that the quantity of turbulent kinetic energy is stronger in the simple cube.

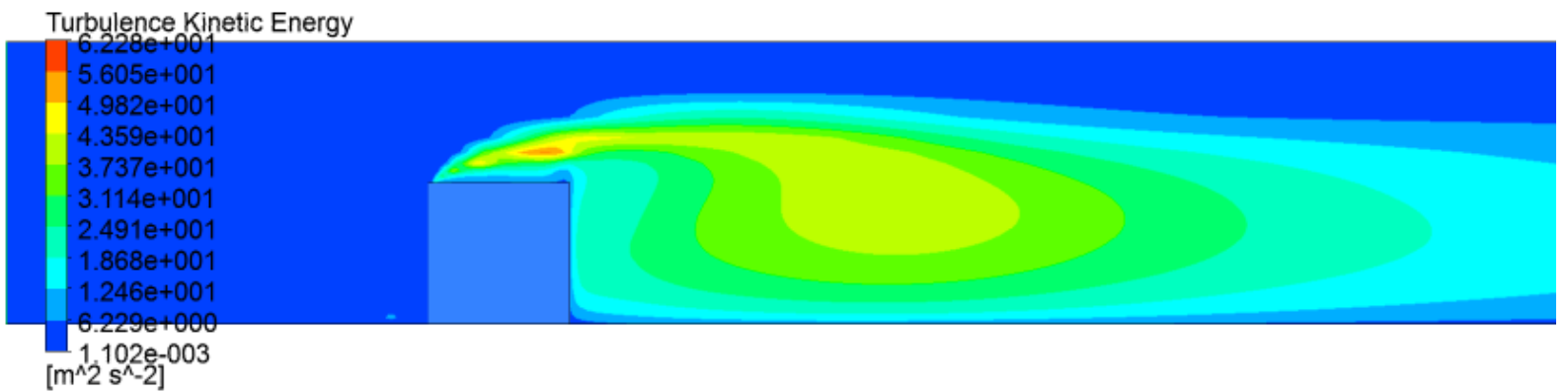

a) Simple cube

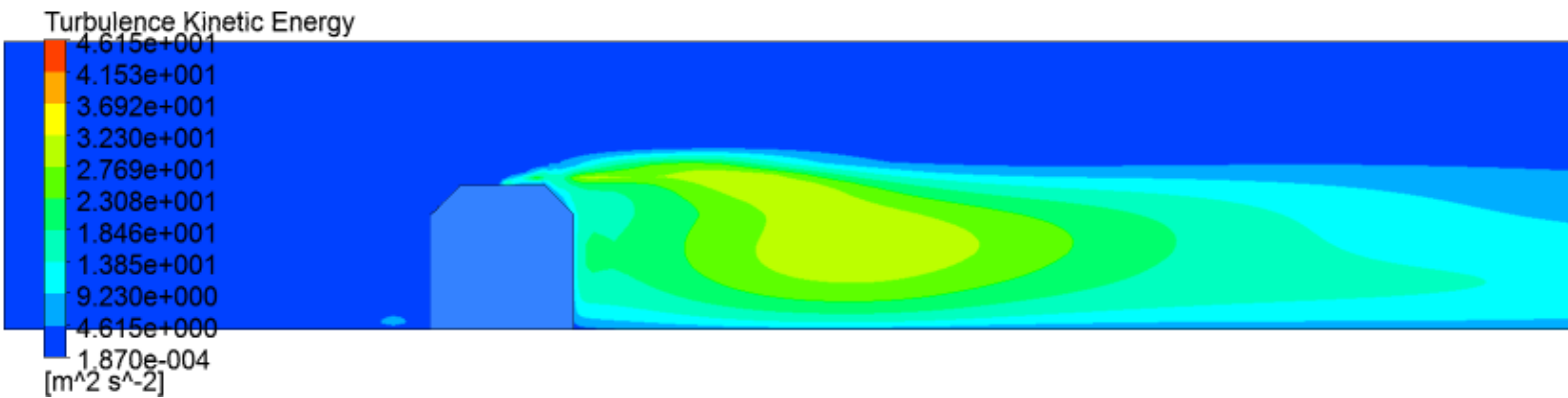

b) Tapered cube

\section{Turbulence Kinetic Energy}

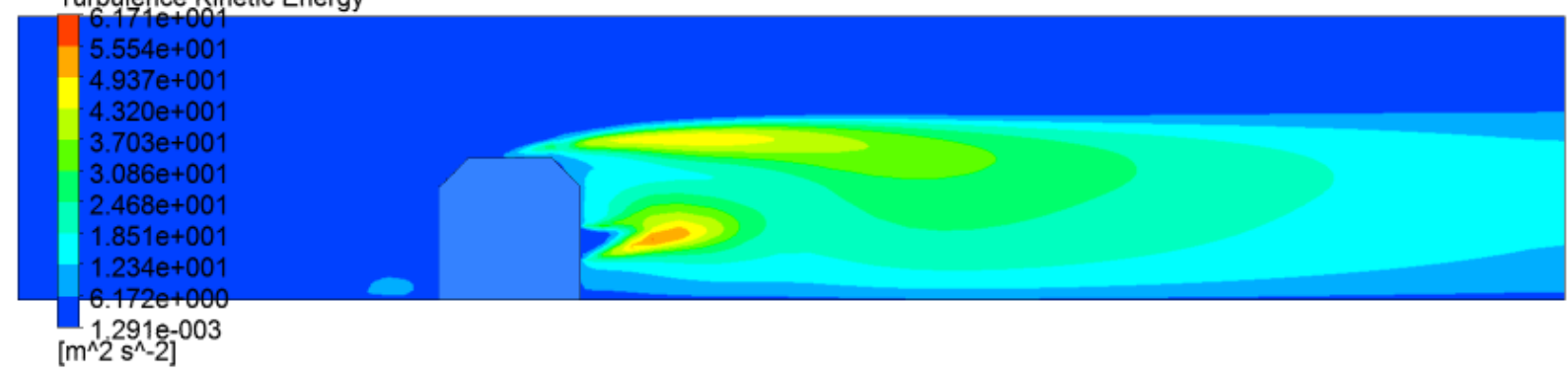

c) Tapered cube with hollow

Figure 7. Contours of Turbulent Kinetic Energy on the symmetry plane for different models of the cube.

For accurate flow analysis and for a good vision of separations and re-attachments on the top of, at the lateral sides and behind the obstacle of the obstacle, the time-averaged streamlines on the symmetry plane and at the floor of the channel for various form of the cube (simple cube, tapered cube and tapered cube with hollow) at Reynolds number $\mathrm{Re}=4 \times 10^{4}$ were presented in (Fig.8) and (Fig.9). In (Fig.8), in the three different configurations, there is a small vortex upstream of the obstacle. Downstream of the cube, we observe the formation of a wide vortex. 
In the case of a tapered dug cube, the vortex emerging from the hollow forms a complex structure and is pen chanting down by the force of the jet. A part of the fluid remains blocked in the region between the tapered upstream edge and the bottom wall of the channel, and above it, the separation is triggered; it is caused by the upstream stopping point of the obstacle which is achieved. Figure.9 and 10 show the 2D and 3D streamlines at the floor of the channel, the blocking effect of the obstacle creates an unfavorable pressure gradient that separates the flow and moves away from the cube, forming a horseshoe vortex. For all forms of the cube, the horseshoe vortex appears upstream and bypasses the obstacle. On the other hand, there is two focus (F), one saddle (S), separation (S) and reattachment (R) point achieved for all configurations that are likewise appearing in the study of R. Martinuzzi and C. Tropea [5]. We note that for the horseshoe the width of the wake is greater in the case of a hollowed-out cube and smaller in the case of the tapered cube.

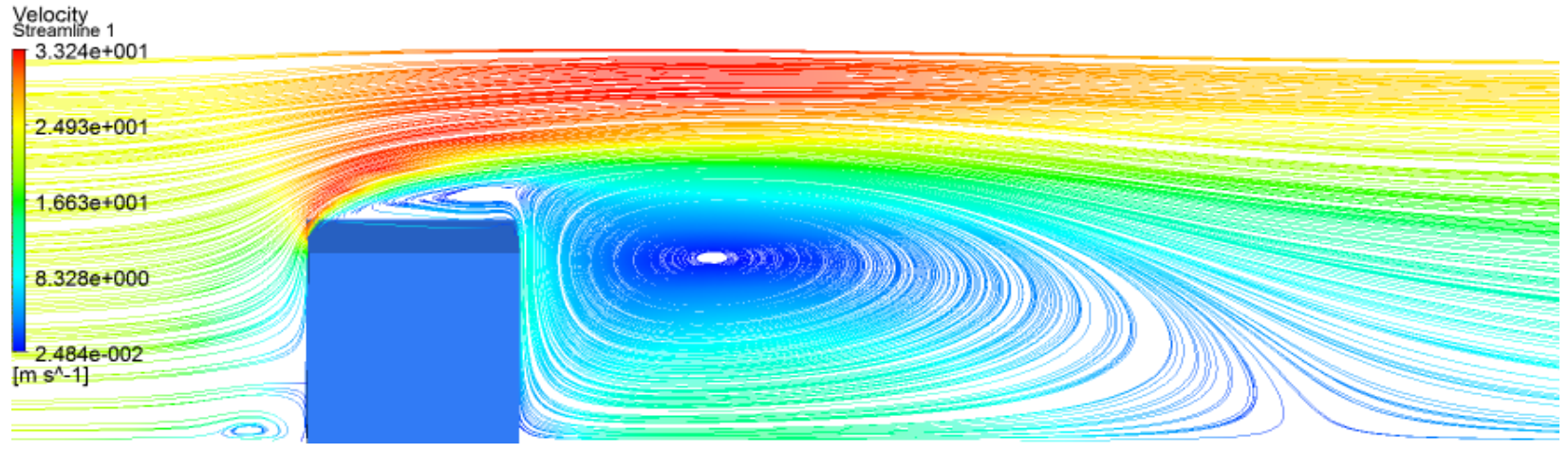

a) Simple cube

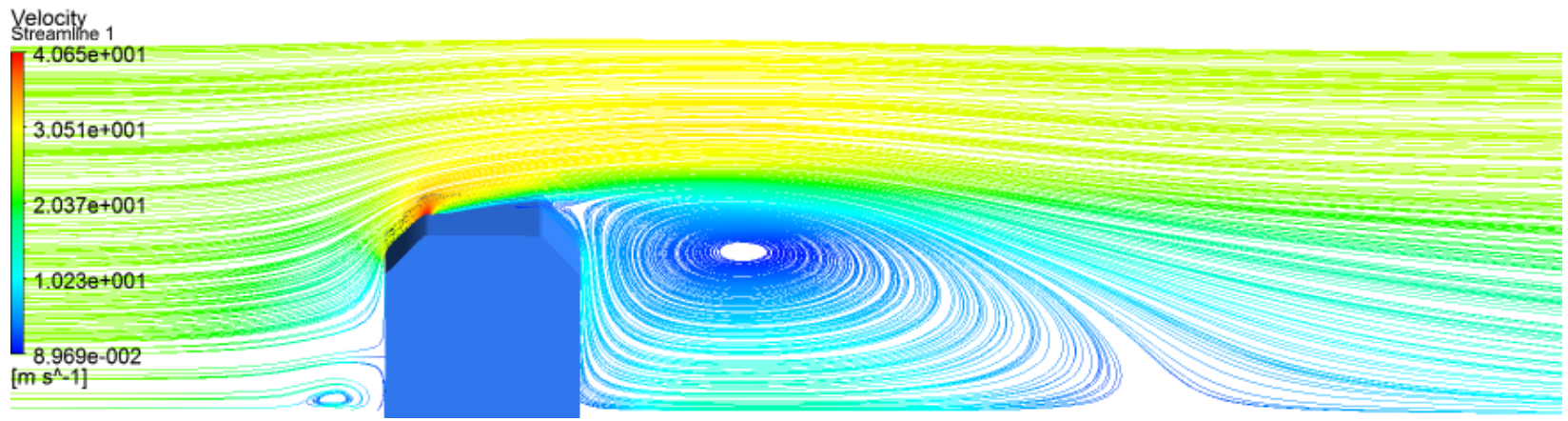

b) Tapered cube

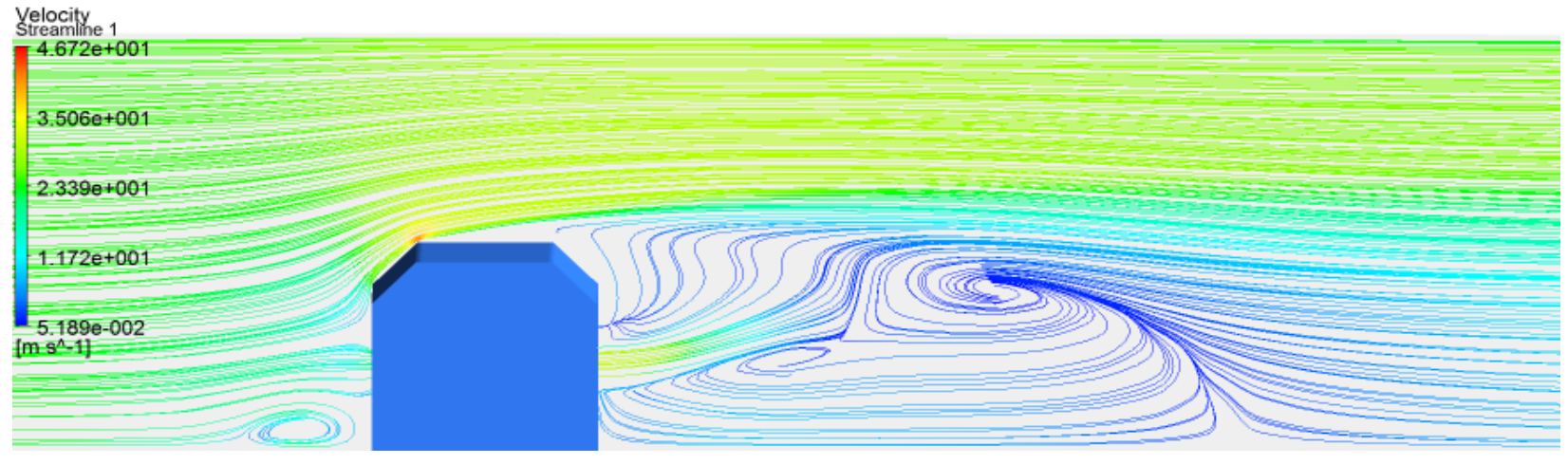

c) Tapered cube with hollow

Figure 8. 2D streamlines at the symmetry plane $(\mathrm{z}=0)$ for a) simple, b) tapered and c) tapered with hollow cubes 


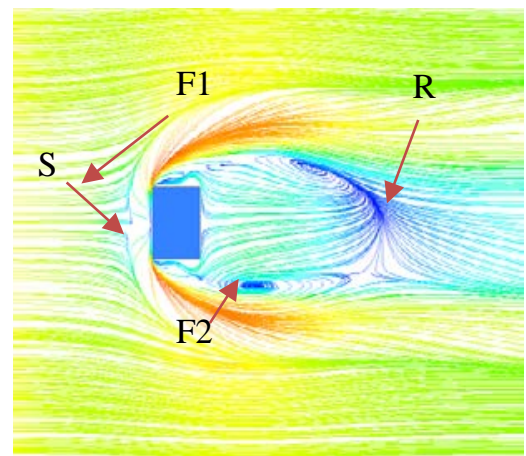

a) Simple cube

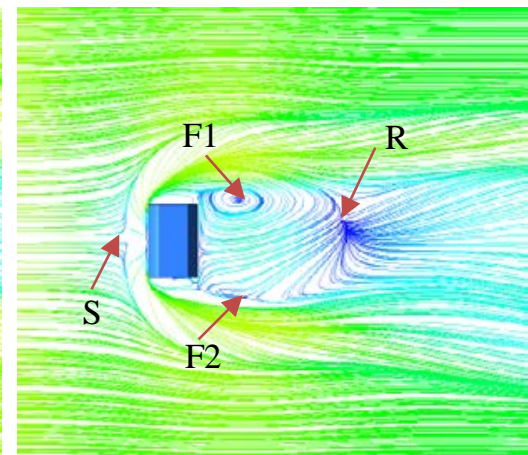

b) Tapered cube

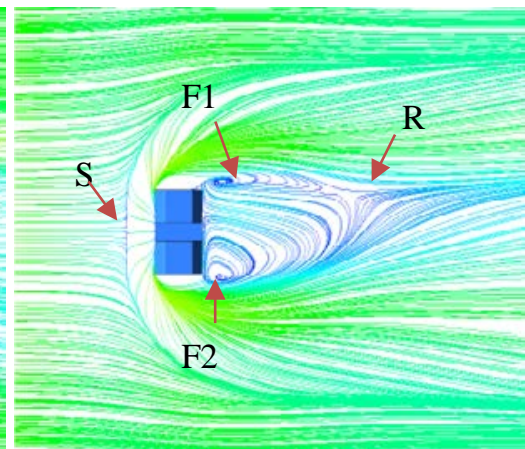

c) Tapered cube with hollow

Figure 9. 2D Streamlines at the floor of the channel at Reynolds number $\mathrm{Re}=4 \times 10^{4}$ for a) simple, b) tapered and c) tapered with hollow cubes

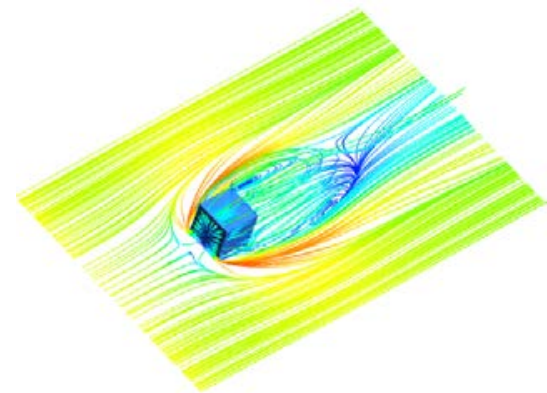

a) Simple cube

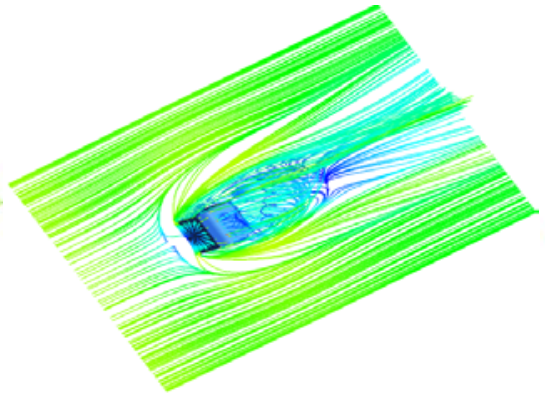

b) Tapered cube

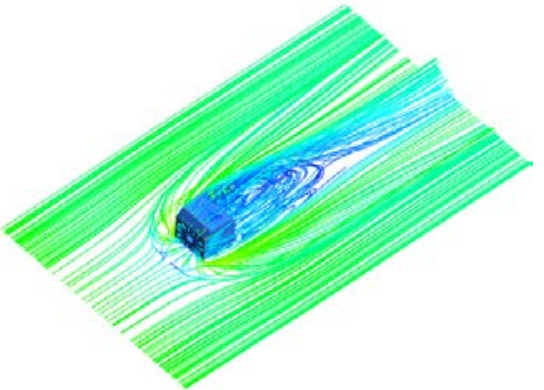

c) Tapered cube with hollow

Figure 10. 3D streamlines at Reynolds number $\mathrm{Re}=4 \times 10^{4}$ for a) simple, b) tapered and c) tapered with hollow cubes

In Fig. 11, trace lines on the surface of the cube presented a steady flow in the high face and lateral sides. A separation of flux is observed at the hollow forming a node. Above the cube and on the high side, the flow is stable until the arrival of the tapered part of the cube; one sees a region of recirculation.
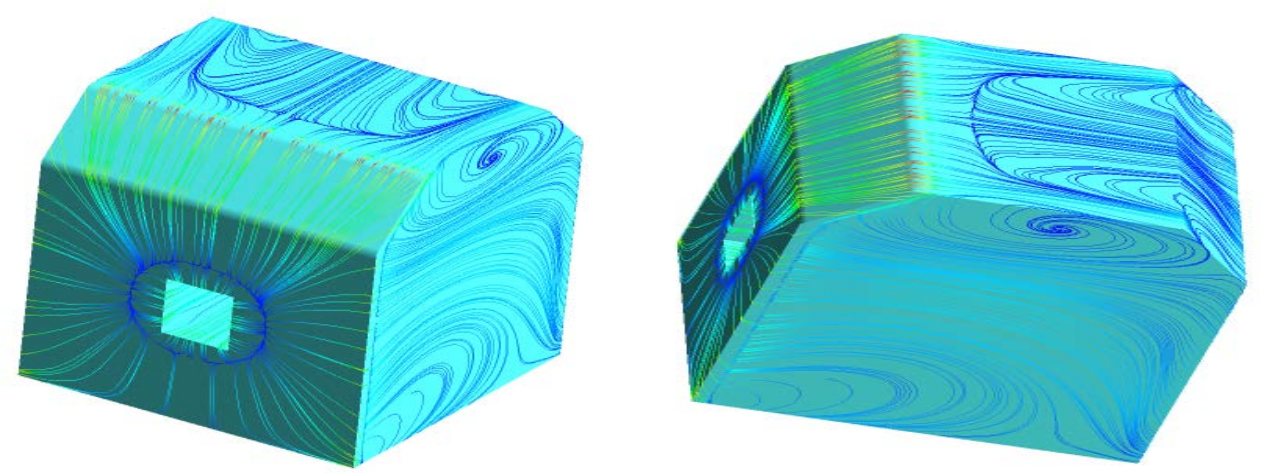

Figure 11.Trace-lines on the surface of the tapered cube with hollow

Figures (12-16) illustrated the velocity profiles (streamwise) at the symmetry plane $(\mathrm{z}=0)$ and different line positions ( $\mathrm{x} / \mathrm{H}=-0.5,0.5,1,1.5$ and 2$)$ respectively upstream, above and downstream the obstacle. The range $\mathrm{y} / \mathrm{H}$ is the meantime $0 \leq \mathrm{y} / \mathrm{H} \leq 2$ for all positions except for the position $\mathrm{x} / \mathrm{H}=0.5$ where $1 \leq \mathrm{y} / \mathrm{H} \leq 2$. For the 5 configurations, there are two recirculation zones: one logarithmic zone due to the main flow, the other is a lower parabolic return zone due to the recirculation vortex. For the first position, upstream the cube $(\mathrm{x} / \mathrm{H}=-0.5)$, we observe that the 3 profiles are almost identical; except close to the wall, we note that of the tapered dug cube is larger than the others. In the case $(\mathrm{x} / \mathrm{H}=0.5)$, the velocity profile for the simple cube is greater than the other cases which are uniform. In 
the middle of the block ( $\mathrm{x} / \mathrm{H}=1)$, the stream wises of the two forms (tapered cube and tapered cube with hollow) are identical from $\mathrm{y} / \mathrm{H}=1$, at the lower end of this value, there is a sharp increase in the axial velocity and this is because it is at the level of the flow inside the cube (in the hollow) and we note that the span of the curve for the tapered cube is larger. Downstream the cube $(\mathrm{x} / \mathrm{h}=1.5)$, we can see two distinct zones, above and below $\mathrm{y} / \mathrm{H}=1$. Above $\mathrm{y} / \mathrm{H}=1$, we note that the velocity profiles for the tapered cube and the tapered cube with hollow are the same and their profiles are smaller than the simple cube, but below the value y / $\mathrm{H}=1$, we observe that the profile of the simple cube and the tapered cube are identical and are smaller than that of the tapered cube with hollow, and we note an abrupt increase in velocity, this is due to the injection at the level close to the hollow. For figure.16, the velocity profiles for the simple cube and the tapered cube are almost identical, so the profile of the tapered cube with hollow is wider.

A presentation of the numerical results of pressure profiles in the direction of flow for three different cube models in the plane of symmetry is given in Figure 17 at the position $\mathrm{x} / \mathrm{H}=2 \mathrm{~m}$ (after the obstacle). The value of the pressure reaches the maximum value of $450 \mathrm{~Pa}$ for the simple cube model, and because of this, the pressure is higher in the simple cube than the others. Besides, the axial pressure decreases sharply because it is located at the level of the flow located behind the cube (exit of the hollow).

It is found that the loss of pressure is relatively low for the case of the obstacle models of the tapered cube and the tapered cube with hollow. On the other hand, this loss of pressure is quite important for the case of the simple cube model.

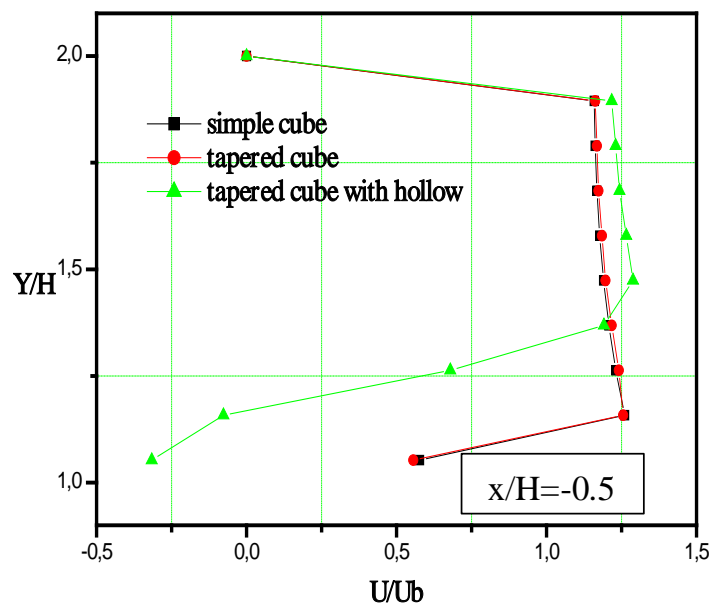

Figure 12. Streamwise velocity profiles at the plane $\mathrm{z}=0$ for $(\mathrm{x} / \mathrm{H}=-0.5)$

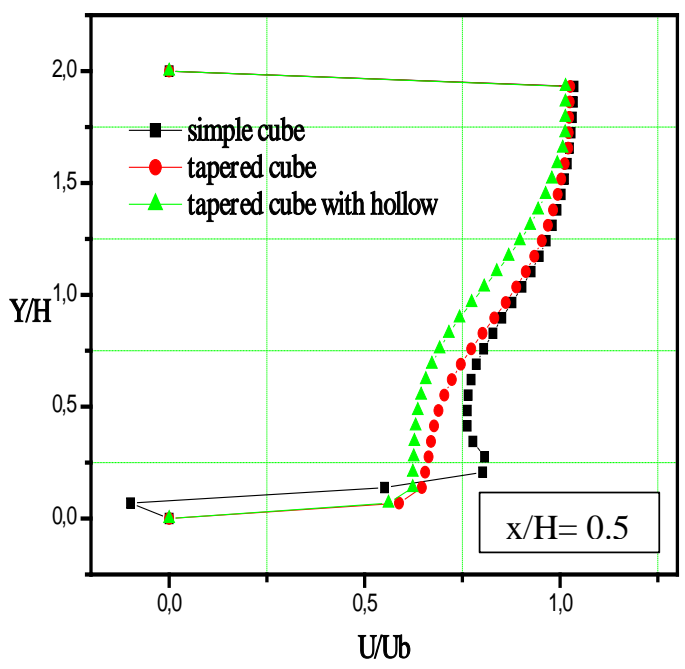

Figure 13. Streamwise velocity profiles at the plane $\mathrm{z}=0$ for $(\mathrm{x} / \mathrm{H}=0.5)$

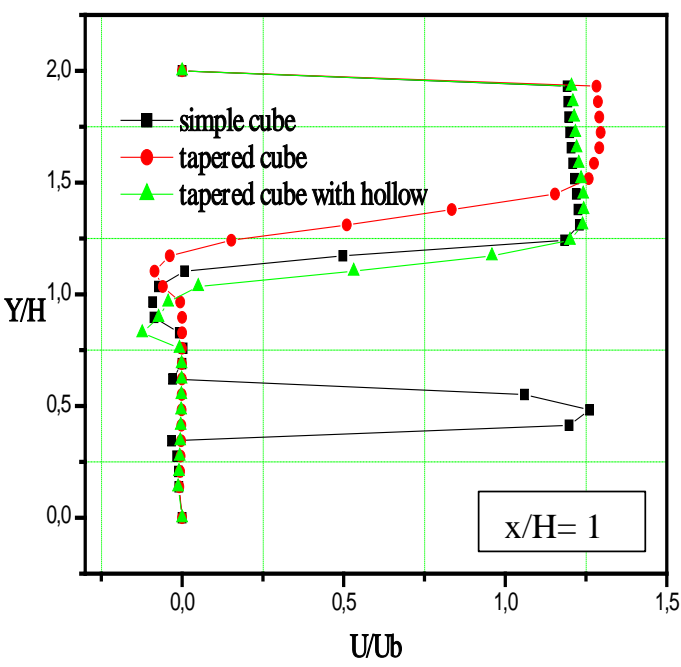

Figure 14. Streamwise velocity profiles at the plane $\mathrm{z}=0$ for $(\mathrm{x} / \mathrm{H}=1)$ 


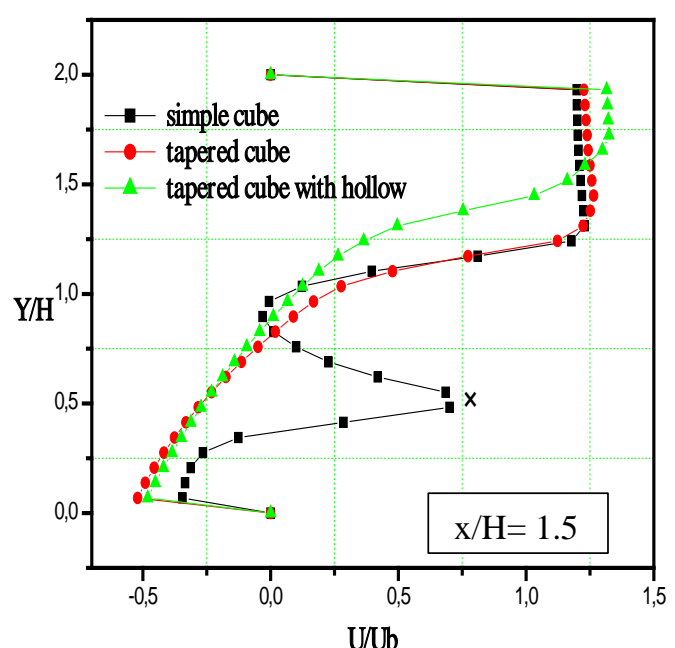

Figure 15. Streamwise velocity profiles at the plane $\mathrm{z}=0$ for $(\mathrm{x} / \mathrm{H}=1.5)$

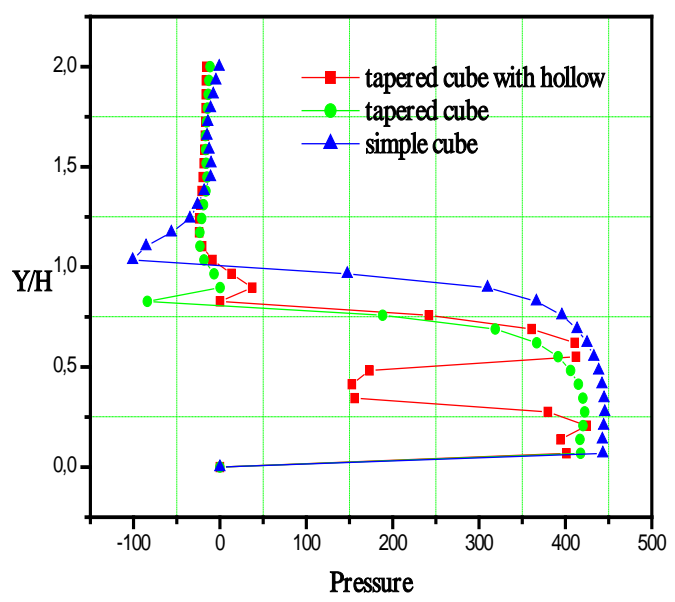

a)

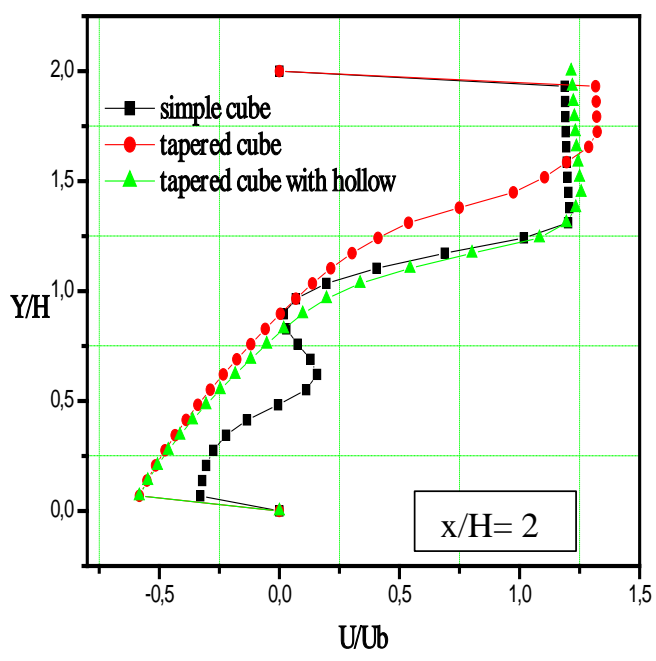

Figure 16. Streamwise velocity profiles at the plane $\mathrm{z}=0$ for $(\mathrm{x} / \mathrm{H}=2)$

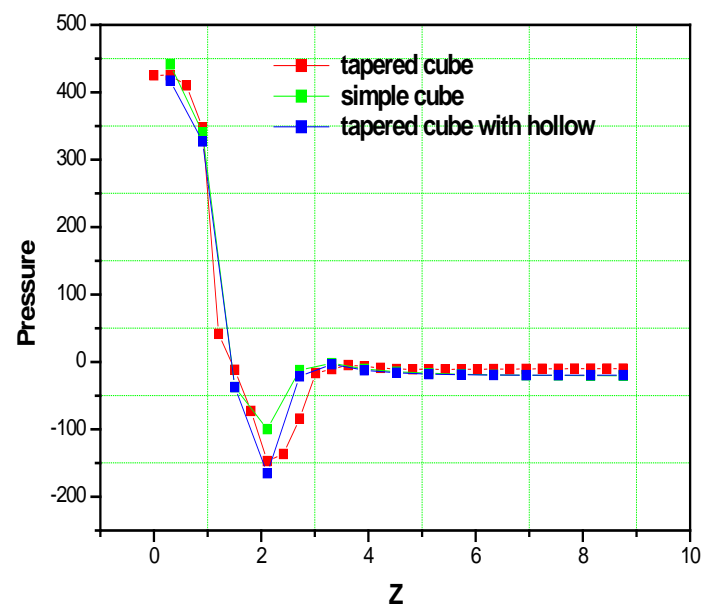

b)

Figure 17. Pressure profiles $a)$ at the plane $\mathrm{z}=0$ for $(\mathrm{x} / \mathrm{H}=0)$ and $\mathrm{b})$ at the plane $\mathrm{y}=0$ for $(\mathrm{x} / \mathrm{H}=0)$

\section{CONCLUSION}

The problem treated in this work is a three-dimensional simulation using the ANSYS CFX calculation code to carry out a three-dimensional numerical simulation of turbulent flow around an obstacle with three different forms (simple cube, a cube with two upper vertices, and tapered dug cube). The flow characteristics of the surface-mounted cube as a function of the Reynolds number of $\mathrm{Re}=4 \times 10^{4}$ have studied with $\mathrm{k}-\omega \mathrm{SST}$ turbulence model. This study has allowed a 3D simulation to analyze and understand some important physical aspects. In this type of flow, especially we tried to emphasize the role of the presence of obstacles in the channel, the distribution of dynamic and thermal exchanges. The analysis of simulation results confirms that:

The quantity of turbulent kinetic energy is stronger in the simple cube. The streamline figures proved the texture of the vortices around the different forms of the block, there appeared three vortices upstream, above and downstream of the obstacle. In the model of the tapered cube with hollow; there was the formation of another vortex downstream of the cube at the outlet of the hollow. For the streamwise velocity, there are two recirculation zones: one logarithmic zone due to the main flow, the other is a lower parabolic return zone due to the recirculation vortex. 
We find that the loss of pressure is relatively low for the case of the obstacle models of the tapered cube and the tapered cube with hollow. On the other hand, this loss of pressure is quite important for the case of the simple cube model.

\section{NOMENCLATURE}

Pr Pranth Number

U Velocity, $\mathrm{m} / \mathrm{s}$

Ub Mean bulk velocity, $\mathrm{m} / \mathrm{s}$

$\mathrm{u}_{\mathrm{i}}{ }^{\prime} \quad$ Time-averaged velocity in $\mathrm{x}_{\mathrm{i}}$ direction, $\mathrm{m} / \mathrm{s}$

$\mathrm{u}_{j}{ }^{\prime} \quad$ Time-averaged velocity in $\mathrm{x}_{\mathrm{j}}$ direction, $\mathrm{m} / \mathrm{s}$

$\mathrm{u}_{\mathrm{i}}{ }^{\prime} \mathrm{u}_{\mathrm{j}}{ }^{\prime} \quad$ Reynolds stresses, $\mathrm{i}, \mathrm{j}=\mathrm{x}, \mathrm{y}, \mathrm{z} \mathrm{m}^{2} / \mathrm{s}^{2}$

$\mathrm{u}, \mathrm{v}, \mathrm{w} \quad$ Next speed components (x, y, z), m/s

$\mathrm{P} \quad$ Pressure, $\mathrm{Pa}$

$\mathrm{T} \quad$ Temperature, $\mathrm{K}$

$\mathrm{P}_{\mathrm{k}} \quad$ Rate of Production

h Canal height, $\mathrm{m}$

$\mathrm{H} \quad$ Height of the obstacle, $\mathrm{m}$

L Channel with, $m$

W Cross-stream With, $m$

Re Reynolds number

$\mathrm{k} \quad$ kinetic energy of turbulence, $\mathrm{m}^{2} / \mathrm{s}^{2}$

Greek symbols

$\phi \quad$ Physical size

$\mu \quad$ Dynamic viscosity, Pa.s

$\mu_{\mathrm{t}} \quad$ Turbulent kinematic eddy viscosity, Pa.s

$\sigma_{k} \quad$ Prandtl number of the turbulent kinetic energy

$\sigma_{\varepsilon} \quad$ Dissipation energy

$\varepsilon \quad$ Turbulent dissipation, $\mathrm{m}^{2} / \mathrm{s}^{3}$

$\rho \quad$ Density, $\mathrm{kg} / \mathrm{m}^{3}$

$\omega \quad$ Specific dissipation, $1 / \mathrm{s}$

\section{REFERENCES}

[1] G. Bitsuamlak, T.Stathopoulos, C.Bedard. Effects of upstream two-dimensional hills on design wind loads: a computational approach. Wind and Structures 2006; 9(1):37-58. http://dx.doi.org/10.12989/was.2006.9.1.037

[2] Nemdili Saleha, Nemdili Fadèla, Azzi Abbès. Improving cooling effectiveness by the use of chamfers on the top of electronic components. Microelectronics Reliability 2015;55(7):1067-1076. https://doi.org/10.1016/j.microrel.2015.04.006

[3] Matthew Hainesa, Ian Taylor. Numerical investigation of the flow field around low rise buildings due to a downburst event using large eddy simulation. Journal of Wind Engineering \& Industrial Aerodynamics 2018;172:12-30. https://doi.org/10.1016/j.jweia.2017.10.028

[4] H.J. Hussein, R.J. Martinuzzi. Energy balance for the turbulent flow around a surface-mounted cube placed in a channel. Phys. Fluids 1996;8764-780. https://doi.org/10.1063/1.868860

[5] R. Martinuzzi, C. Tropea. The flow around a surface-mounted prismatic obstacle placed in a fully developed channel flow, J.Fluids Eng. 1993;115:85-92. https://doi.org/10.1115/1.2910118

[6] JongYeon Hwang, KyungSoo Yang. Numerical study of vertical structures around a wall-mounted cubic obstacle in channel flow. Physics of Fluids 2010;16(7):2382-2394. https://doi.org/10.1063/1.1736675

[7] German Filippini, Gerado Franck, Norberto Nigro. Large Eddy Simulations of the flow around a square cylinder. Mecanica Computacional 2005;24:1279-1298. https://doi.org/10.1016/j.jfluidstructs.2020.103107

[8] Hee Chang Lim, T.G. Thomas, Ian P. Castro. Flow around a cube in a turbulent boundary layer: LES and experiment. Journal of Wind Engineering and Industrial Aerodynamics 2009;97(2):96-109. https://doi.org/10.1016/j.jweia.2009.01.001

[9] S. Krajnovi'c, L. Davidson. Large-eddy simulation of the flow around a bluff body. AIAA Journal 2002;40(5):927-936. https://doi.org/10.2514/2.1729 
[10] Dan Gu, Hee Chang Lim. Wind flow around rectangular obstacles and the effects of aspect ratio. The Seventh International Colloquium on Bluff Body Aerodynamics and Applications (BBAA7) 2012;2-6.

[11] Becker, H. Lienhart, F. Durst. Flow around three-dimensional obstacles in boundary layers, J. Wind Eng. Ind. Aerodyn. 2002;90:265-279.

[12] Alexander Yakhot, Heping Liu, Nikolay Nikitin. Turbulent flow around a wall-mounted cube: A direct numerical simulation. International Journal of Heat and Fluid Flow 2006;27(6):994-1009. https://doi.org/10.1016/j.ijheatfluidflow.2006.02.026

[13] B.Rostane, K.Aliane. Three Dimensional Simulation for Turbulent Flow Around Prismatic Obstacle with Rounded Downstream Edge Using the k- $\omega$ SST Model. International Review of Mechanical Engineering (I.RE.M.E.) 2015;9(3):1970-8734.

[14] SARI-HASSOUN Zakaria, ALIANE Khaled. Simulation numérique de l'écoulement turbulent autour d'obstacles a arête amont courbée. International Journal of Scientific Research \& Engineering Technology (IJSET) 2016;196-201.

[15] Aliane, K. Passive control of the turbulent flow over a surface-mounted rectangular block obstacle and a rounded rectangular obstacle. International Review of Mechanical Engineering (IREME) 2011;5(2);305314.

[16] Mohd ARIFF, Salim M. SALIM, and Siew Cheong CHEAH, « Wall y+ approach for dealing with turbulent flow over a surface-mounted cube: part 2 - high Reynolds number », Seventh International Conference on CFD in the Minerals and Process Industries CSIRO, Melbourne, Australia 9-11 December 2009.

[17] Karima HEGUEHOUG ep BENKARA-MOSTEFA, Zoubir NEMOUCHI, Farid GACI, «Contribution à l'étude de l'écoulement Tridimensionnel turbulent autour d'un profil et à travers une série d'aubes fixes», TERMOTEHNICA 1/2010.

[18] Merahi, M. Abidat, A. Azzi, O. Hireche. Numerical assessment of incidence losses in an annular blade cascade. Séminaire international de Génie Mécanique. Sigma’02 ENSET. Oran. 28 \& 29 April 2002.

[19] Mohammed Amine Amraoui, Khaled Aliane, 'Three-dimensional Analysis of Air Flow in a Flat Plate Solar Collector”, Periodica Polytechnica Mechanical Engineering 62(2), pp. 126-135, 2018. https://doi.org/10.3311/PPme.11255

[20] Sercan Dogan, Sercan Yagmur, Ilker Goktepeli, and Muammer Ozgoren. Assessment of Turbulence Models for Flow around a Surface-Mounted Cube. International Journal of Mechanical Engineering and Robotics Research 2017;6(3). doi: 10.18178/ijmerr.6.3.237-241

[21] Kanfoudi, H., G. Bellakhall, M. Ennouri, A. Bel Hadj Taher and R. Zgolli. Numerical Analysis of the Turbulent Flow Structure Induced by the Cavitation Shedding Using LES. Journal of Applied Fluid Mechanics. 2017;10(3):933-46. DOI: 10.18869/acadpub.jafm.73.240.27384

[22] Seyed Reza Djeddi, Ali Masoudi, Parviz Ghadimi. Numerical Simulation of Flow around Diamond-Shaped Obstacles at Low to Moderate Reynolds Numbers. American Journal of Applied Mathematics and Statistics 2013;1(1):11-20. DOI:10.12691/ajams-1-1-3

[23] Liakos, A. and Malamataris, N.A. Direct numerical simulation of steady-state, three dimensional, laminar flow around a wall-mounted cube, Physics of Fluids 2014;26(5):053603. https://doi.org/10.1063/1.4876176

[24] Diaz-Daniel, C., Laizet, S., \& Vassilicos, J. (2017). Direct Numerical Simulations of a wall-attached cube immersed in laminar and turbulent boundary layers. Preprint submitted to the International Journal of Heat and Fluid Flow 2017;68:269-280. https://doi.org/10.1016/j.ijheatfluidflow.2017.09.015

[25] Sumner, D., Rostamy, N., Bergstrom, D., \& Bugg, J. Influence of aspect ratio on the flow above the free end of a surface-mounted finite cylinder. International Journal of Heat and Fluid Flow 2015;56:290-304. https://doi.org/10.1016/j.ijheatfluidflow.2015.08.005

[26] Sumner, D., Rostamy, N., Bergstrom, D., \& Bugg, J. D. Influence of aspect ratio on the mean flow field of a surface-mounted finite-height square prism. International Journal of Heat and Fluid Flow 2017;65:1-20. https://doi.org/10.1016/j.ijheatfluidflow.2017.02.004

[27] Siddhesh Shinde, Eric Johnseny and Kevin Makiz, (2017). Understanding the effect of cube size on the near wake characteristics in a turbulent boundary layer. 47th AIAA Fluid Dynamics Conference, Denver, Colorado 2017;3640. https://doi.org/10.2514/6.2017-3640

[28] M. Ennouri, H. Kanfoudi, A. Bel Hadj Taher and R. Zgolli, (2019). Numerical Flow Simulation and Cavitation Prediction in a Centrifugal Pump using an SST-SAS Turbulence Model. Journal of Applied Fluid Mechanics 2019;12(1):25-39. DOI: 10.29252/jafm.75.253.28771

[29] Sari Hassoun Zakaria, Aliane Khaled, Henaoui Mustapha. Experimental Study of a Flat Plate Solar Collector Equipped with Concentrators. International Journal of Renewable Energy Research (IJRER), 2017;7(3):1028-1031. 
Journal of Thermal Engineering, Research Article, Vol. 7, No. 2, Special Issue 13, pp. 256-269, February, 2021

[30] M. Bayareh and A. Nourbakhsh. Numerical simulation and analysis of heat transfer for different geometries of corrugated tubes in a double pipe heat exchanger. Journal of Thermal Engineering 2019;5(4):293-301. https://doi.org/10.18186/thermal.581775

[31] F. Menter. Two-Equation Eddy-Viscosity. Turbulence Models for engineering Application. AIAA Journal, 1994;32:1598-1605. https://doi.org/10.2514/3.12149

[32] D. C. Wilcox. Turbulence Modeling for CFD Second Edition, D.C.W. Industries, (1998).

[33] W. Jones and B. Launder. The calculation of low-Reynolds-number phenomena with a two-equation model of turbulence. International Journal of Heat and Mass Transfer 1973;16(6):1119-1130. https://doi.org/10.1016/0017-9310(73)90125-7 